\title{
Téoros
}

Revue de recherche en tourisme

\section{Le tourisme des gais et des lesbiennes}

Vers un Village global ?

\section{Alain Caron et Lucie K. Morisset}

Volume 19, numéro 2, été 2000

Le tourisme des gais et des lesbiennes

URI : https://id.erudit.org/iderudit/1071956ar

DOI : https://doi.org/10.7202/1071956ar

Aller au sommaire du numéro

Éditeur(s)

Université du Québec à Montréal

ISSN

0712-8657 (imprimé)

1923-2705 (numérique)

Découvrir la revue

Citer ce document

Caron, A. \& Morisset, L. K. (2000). Le tourisme des gais et des lesbiennes : vers un Village global ? Téoros, 19(2), 3-5. https://doi.org/10.7202/1071956ar d'utilisation que vous pouvez consulter en ligne.

https://apropos.erudit.org/fr/usagers/politique-dutilisation/ 


\title{
LE TOURISME DES GAIS ET DES LESBIENNES
}

\author{
VERS UN VILLAGE GLOBAL?
}

\section{Alain Caron, rédacteur invité ${ }^{~}$ et Lucie K. Morisset}

La pertinence d'un numéro de la revue Téoros consacré aux voyages des gais et des lesbiennes repose sur un double constat. D'une part, le simple intérêt économique, qu'appuie un nombre croissant d'études, veut que les gais ${ }^{2}$ et les lesbiennes représentent un "marché » de choix parce qu'ils voyagent davantage. Il est vrai que, souvent moins contraints par des responsabilités familiales ${ }^{3}$, ils peuvent consacrer une grande part de leurs revenus aux loisirs, tout en jouissant d'un horaire plus flexible et, partant, d'une grande mobilitét ${ }^{4}$. D'autre part, alors que la popularité de certaines destinations dites « gaies » influe dorénavant même sur la carte des voyages à l'échelle internationale, les visiteurs gais et lesbiens de nombreux pays sont souvent victimes d'un accueil mitigé au sein des infrastructures touristiques, voire de harcèlement de la part des forces policières. Le présent numéro de Téoros vise à présenter un bilan de cette situation paradoxale qu'il faudra bien, un jour, aborder par-delà la mythification dont le fameux «argent rose » est l'objet.

Il n'est donc pas innocent que les articles que nous avons colligés ici abordent, pour plusieurs, le paradoxe des « destinations ouvertes » et des « destinations fermées ». Certes, comme le présente Dino Starcevic dans le cas du Costa Rica, des communautés gaies et lesbiennes se sont organisées au cœur de zones d'emblée peu tolérantes ; il n'en reste pas moins - c'est ce que rapporte l'article de Roberto Jovel, d'Amnistie Internationale - que la situation des gais et des lesbiennes est toujours alarmante dans plusieurs régions du monde, limitant dans les faits la mobilité des voyageurs gais et lesbiens. Et pourtant... cette mobilité demeurerait l'un des facteurs dominants de l'imaginaire collectif des homosexuels : Line Chamberland, dans son article sur la « recherche de soi, recherche d'un ailleurs », met en exergue ce phénomène de la définition d'une identité lesbienne, qui n'est pas sans évoquer la recherche de quelque pays d'Oz, «somewhere over the rainbow ${ }^{5} » . . . \mathrm{De}$ fait, que la référence au célèbre film soit commune parmi les gais n'est pas qu'une coïncidence : si homosexualité suppose mobilité, l'exclamation de l'héroïne du Wizard of $\mathrm{Oz}$, «It ain't Kansas anymore », trouve écho dans l'engouement des gais et des lesbiennes pour les grands centres urbains.

En marge d'enclaves disséminées de par le monde - on peut penser à Provincetown, par exemple - , hors des villes, auprès de stations balnéaires favorisées, notamment, les grandes villes deviennent ainsi des destinations privilégiées : s'y trouvent ces «Villages gais » dont les atmosphères bien typées, d'ailleurs, retiennent aussi un nombre croissant de visiteurs hétérosexuels. Mais les « Villages » sont eux-mêmes des objets de controverse : tandis que Frank Remiggi, dans l'article «Homosexualité et espace urbain...», retrace l'évolution de cette personnalité géographique qu'est le Village gai de Montréal, Julie Podmore analyse le parcours lesbien montréalais qui, justement, trouve son expression non pas dans la relative uniformité du Village gai, territoire de consommation des hommes, mais dans la pluralité de l'espace interstitiel du boulevard Saint-Laurent.

De l'autre côté de telles consommations, c'est-à-dire chez les «producteurs » de l'offre touristique, les interrogations appellent aussi au paradoxe : les incontournables tabous, partiellement responsables de l'état lacunaire des recherches sur le tourisme des gais et des lesbiennes, ne sont pas étrangers aux vacillantes définitions d'un « segment de marché » que l'approche clientèle aimerait bien cerner. Devant

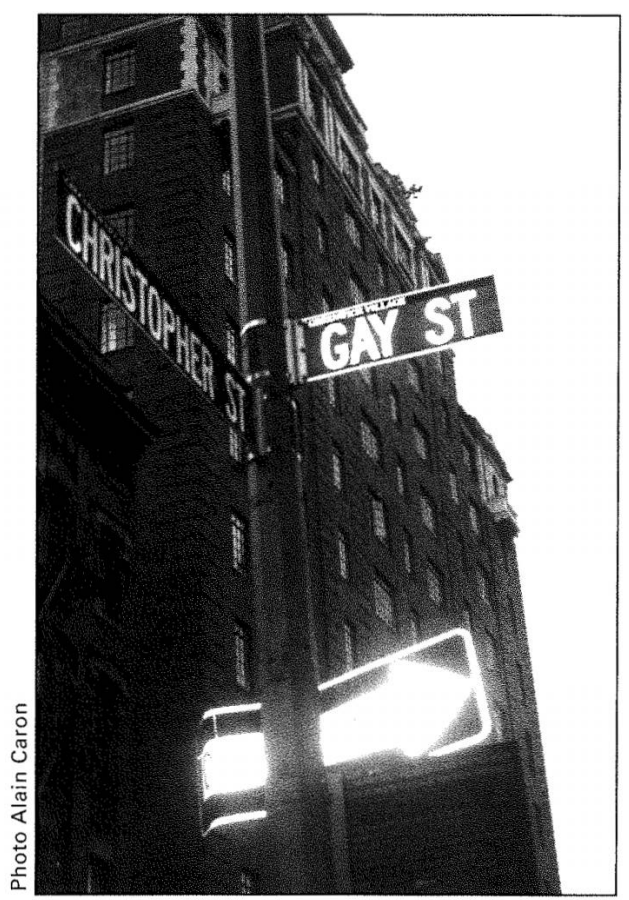

La toponymie des rues de Greenwich Village (New York) reflète la présence d'une importante communauté gaie et lesbienne. 
la question « que cherchent ces gais et ces lesbiennes, hormis leurs semblables ou, à tout le moins, l'acceptation que ce voisinage sous-entend? ", sans doute faut-il d'emblée écarter la thèse d'un produit unique qui fédérerait les intérêts d'un groupe qu'on imagine encore souvent homogène en ce qu'il pratiquerait des activités « contre nature » (!). Assimiler bêtement le tourisme des gais et des lesbiennes au tourisme sexuel - partant, à la prostitution et à la pédophilie - reviendrait à croire, par exemple, que le tourisme social destiné aux communautés défavorisées se définirait par la quête unanime de ces communautés d'être défavorisées ${ }^{6} .$. Évidemment, la dimension sexuelle importante de la culture gaie ne peut être évacuée du tableau problématique, non plus que ne devrait l'être la sexualité hétérosexuelle du tourisme en général; mais la trilogie "Profit, Sex and Identity » qu'explore Howard Hughes dans ce numéro, si elle nourrit l'association du segment de marché au groupe identitaire, ne devrait pas pour autant masquer d'autres facettes du rapport des gais et des lesbiennes au voyage.

Une chose est certaine, néanmoins : la question identitaire qui détermine puissamment ce rapport influe tout autant sur les motifs des voyages gais et lesbiens que sur la configuration imaginaire des destinations elles-mêmes : tout se passe comme si la « métamorphose de la société d'accueil » tant annoncée par les sociologues du tourisme prenait ici de nouvelles proportions. Les fêtes bénéfice ou circuit parties, fréquentées en masse par ces hommes gais adeptes des salles de musculation dont les médias ressassent les images, attirent une attention croissante qui témoigne de ce phénomène : populaires chez les jeunes adultes, de telles fêtes sont aussi devenues un espace de rapprochement des communautés homosexuelles et hétérosexuelles autour, nommément, de la cause universelle du sida. Sans être exclusivement responsables de la création des destinations, des événements de ce type, que présente ici Caroline Rousse, sont associés à des retombées économiques appréciables ; pour les communautés gaies et lesbiennes, des fêtes de la Fierté lesbienne, gaie, bisexuelle, travestie et transsexuelle, comme celle qu'organise Divers/ Cité, à Montréal, offrent aussi une occasion de rapprochement. De tels événements attirent une clientèle touristique gaie et lesbienne fort variée, ainsi qu'un nombre record de participants, indépendamment de leur orientation sexuelle ; cela, à nouveau, témoigne du désir de communiquer qui semble ressortir parmi les motivations des gais et des lesbiennes qui voyagent (en fait, la seule existence de destinations spécifiques devrait suffire à attester de la force de cette motivation). Plus encore, cependant, la popularité et la médiatisation des diverses fêtes associées à la communauté gaie et lesbienne, ainsi que la « diaspora » des destinations qu'évoquent les articles de ce numéro de Téoros, évoquent un lien entre le tourisme des gais et des lesbiennes et un nouveau tourisme communautaire, fondé sur la volonté de créer des liens. De là à voir s'esquisser par-delà les frontières un « village global », il n'y a qu'un pas qui paraît déjà, partiellement à tout le moins, franchi : en arrière-plan du drapeau arc-en-ciel ${ }^{7}$, symbole d'homosexualité et de diversité, peut-on voir dans le tourisme des gais et des lesbiennes le miroir des espoirs communautaires inassouvis d'une société occidentale qui continue pourtant de décrier la «mondialisation »?

Il est possible, en effet, que la présence croissante du tourisme des gais et des lesbiennes dans la définition des destinations ne soit qu'un autre visage de la reconfiguration des collectivités autour de la notion d'individu : la facilité qu'ont apparemment gais et lesbiennes de recréer un univers identitaire à l'étranger se révélerait alors un modèle quant à la mise en place de nouveaux espaces de socialisa- tion. Pourtant, à mi-chemin entre une rectitude politique alimentée par les causes associées aux manifestations de la présence homosexuelle et une acceptation sincère, elle-même motivée par le désir de socialiser, le marché et les communautés hôtes hésitent encore, bien que les implications culturelles du tourisme des gais et des lesbiennes, dans les villes notamment, sautent déjà aux yeux.

Cependant, pendant que de nombreuses publications fondent encore la mise en tourisme des destinations gaies sur les bars et les saunas et que des organisations, plus nombreuses encore, utilisent le drapeau arc-en-ciel comme l'image de marque de leur ouverture d'esprit (!), des associations comme l'IGLTA (International Gay and Lesbian Travel Association) regroupent un nombre croissant d'entreprises intéressées à servir gais et lesbiennes ; au Québec, Direction Gay Québec (voir l'article « Tourisme gai et Internet ») vise, semblablement, à étendre la quantité et la qualité de « l'hospitalité gaie ». Et plus encore, le groupe Community Marketing de San Francisco (voir article « Repenser le marketing gai et lesbien... ») a développé une approche plus globale de la culture gaie et lesbienne basée sur l'esprit communautaire et l'insertion dans la société.

Ce numéro de Téoros participe de cet esprit, en vue de poser les bases d'une interface intelligente entre la communauté homosexuelle et les entreprises touristi-

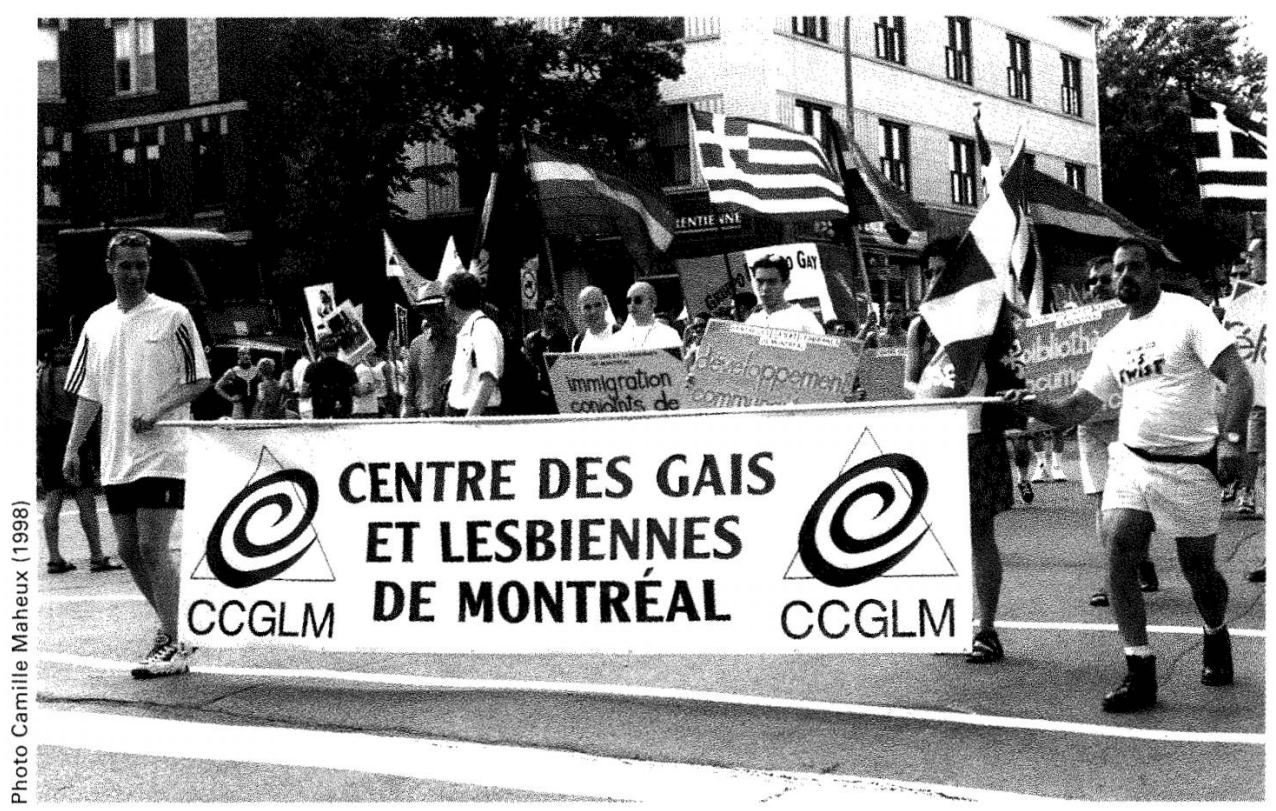

Le défilé organisé par Divers/Cité à Montréal permet de faire connaître les nombreux groupes communautaires gais et lesbiens. 
ques. Ce n'est pas un hasard que cette « revue internationale de recherche en tourisme », basée à Montréal, se soit enfin penchée sur le tourisme des gais et des lesbiennes: le nombre de références montréalaises que ce numéro rassemble, ainsi que le développement de ressources spécifiques au sein de Tourisme Montréal, qui, en partenariat avec la Chambre de commerce gaie du Québec et Tourisme Québec, est devenu pionnier en la matière (Pierre Bellerose et Jean-François Perrier en font ici état), constituaient à nos yeux les prémisses de ce qui pouvait, à tout le moins, se révéler un « cas » intéressant. Il était en effet aussi, parmi nos objectifs, d'exposer les pistes de recherches nombreuses que le tourisme des gais et des lesbiennes recèle : de telles pistes, comme celles que présente Philippe Connil dans ce numéro, nous paraissent porteuses du redéploiement des analyses, que nous appelons de nos vœux. De fait, en arrièreplan du tourisme des gais et des lesbiennes, segment de marché, village global ou construction identitaire, selon les intérêts de chacun, paradoxes et controverses, destinations et motivations, nous sont apparus comme la « pointe de l'iceberg» d'un phénomène plus vaste : connaître mieux ce tourisme conditionnerait peutêtre un choix de société en faveur d'un Tourisme qui, finalement, soit véritablement un outil de développement culturel.

Secrétaire de rédaction de la revue Téoros, Alain Caron s'intéresse au foisonnement des identités qui forgent nos paysages concrets et imaginaires ainsi qu'aux mutations que ces identités subissent lorsqu'elles voyagent et sont confrontées à celles de l'Autre. Il complète actuellement un doctorat en études urbaines à l'Université du Québec à Montréal.

Lucie K. Morisset est professeure au Département d'études urbaines et touristiques de l'UQAM et directrice de la revue Téoros.

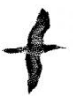

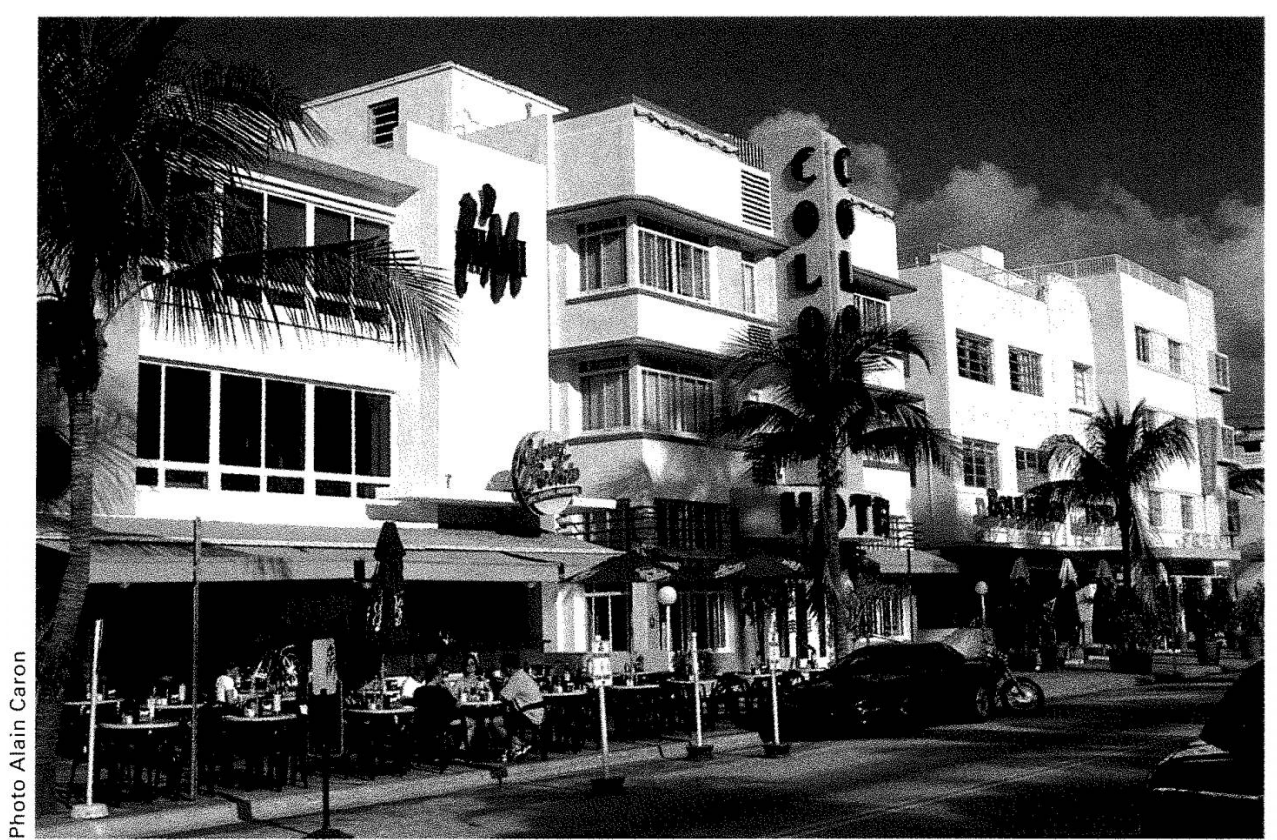

Le quartier Art Déco de Miami Beach (South Beach), summum du design, est une destination gaie de prédilection.

\section{NOTES}

1 Le rédacteur invité tient à souligner la participation essentielle de Philippe Connil à l'élaboration de ce numéro et à la recherche des auteurs qui y ont contribué.

2 Le terme « gai » est reconnu par l'Office de la langue française et utilisé depuis 1952. Identifiant au départ la réalité des homosexuels masculins, on a souvent étendu son sens à la communauté gaie et lesbienne. L'Office reconnaît la forme " gay », directement empruntée à l'étasunien, utilisée de façon invariable, comme c'est la norme dans les autres pays francophones. Il recommande en outre, pour le contexte québécois, d'utiliser la version francisée du terme, « gai », et de l'accorder en genre et en nombre. Au Québec, on a généralement cessé d'utiliser la forme anglophone au début des années 1980.

3 Il est essentiel cependant de noter qu' au sein même de la communauté gaie et lesbienne, il existe une grande disparité des revenus et que plusieurs gais et lesbiennes ont aussi la responsabilité d'enfants. De surcroît, le rapport des salaires des hommes et des femmes est le même pour l'ensemble de la société, peu importe les orientations sexuelles de chacun, ce qui évidemment marginalise les couples lesbiens de l'image du golden boy, certes véhiculée par la portion la plus visible de la communauté gaie, mais qu'il convient néanmoins, à l'évidence, de relativiser.

4 Il n'est d'ailleurs pas impossible - des recherches pourraient être menées dans cette voie - que, hormis la recherche de virilité, ce phénomène de mobilité et de liberté face à la normativité sociale contribue à nourrir le fantasme bien connu des marins dans la communauté gaie.

5 Dans le film The Wizard of $\mathrm{Oz}$ (Le magicien d'Oz), réalisé en 1939 d'après l'œuvre de Frank Baum, une jeune fille du Kansas, Dorothy, incomprise par son entourage, fuit vers un monde imaginaire situé « somewhere over the rainbow ». Au long de son périple, elle rencontre de nombreux êtres qui partagent sa quête et qui finalement, comme elle, trouvent en eux-mêmes ce qu'ils ont cherché en vain loin de leur origine. Cette métaphore avec le tourisme des gais et des lesbiennes - qu'on ne peut évidemment relier à quelque « sens caché » de l'œuvre, qui a d'ailleurs connu plusieurs autres interprétations - est à ajouter au nombre des explications de la mythification de ce film dans la culture gaie nord-américaine.

6 Le rédacteur invité remercie Katia Petkova d'avoir partagé avec lui sa réflexion à ce sujet, qui aurait peut-être requis à lui seul un numéro entier.

7 La communauté gaie et lesbienne a déjà son emblème, d'ailleurs de plus en plus reconnu par les hétérosexuels : il s'agit du drapeau arborant six des couleurs de l'arc-en-ciel (rouge, orangé, jaune, vert, bleu et violet). Une entreprise qui le déploie signale qu'elle est exploitée par des gais ou des lesbiennes ou qu'elle leur est favorable ( $g$ ay friendly). Conçu comme symbole de la diversité, le drapeau a été créé par un artiste de San Francisco à la fin des années 1970. 